\title{
ALCALOIDES $\beta$-INDOLOPIRIDOQUINAZOLÍNICOS DE Esenbeckia grandiflora MART. (RUTACEAE)
}

\author{
Ana Helena Januário*, Paulo Cezar Vieira, Maria Fátima das Graças Fernandes da Silva e João Batista Fernandes \\ Departamento de Química, Universidade Federal de São Carlos, CP 676, 13565-905 São Carlos -SP, Brasil \\ Jorge José de Brito Silva e Lucia Maria Conserva \\ Instituto de Química e Biotecnologia, Universidade Federal de Alagoas, 57072-970 Maceió - AL, Brasil
}

Recebido em 29/7/08; aceito em 29/4/09; publicado na web em 6/10/09

\begin{abstract}
INDOLOPYRIDOQUINAZOLINE ALKALOIDS FROM Esenbeckia grandiflora MART. (RUTACEAE). The chemical composition of two specimens of Esenbeckia grandiflora, collected in the south and northeast regions of Brazil, was investigated. In this study, three $\beta$-indolopyridoquinazoline alkaloids from the leaves (rutaecarpine, 1-hydroxyrutaecarpine) and roots (euxylophoricine D) were isolated for the first time in this genus. In addition, the triterpenes $\alpha$-amyrin, $\beta$-amyrin, $\alpha$-amyrenonol, $\beta$-amyrenonol, $3 \alpha$-hydroxyursan-12-one, and $3 \alpha$-hydroxy-12,13-epoxy-oleanane, the coumarins auraptene, umbelliferone, pimpinelin, and xanthotoxin, the furoquinoline alkaloids delbine and kokusaginine, and the phytosteroids sitosterol, stigmasterol, campesterol and $3 \beta-O-\beta-D-$ glucopyranosylsitosterol were also isolated from the leaves, twigs, roots and stems of this species. Structures of these compounds were established by spectral analysis.
\end{abstract}

Keywords: Esenbeckia grandiflora; Rutaceae; indolopyridoquinazoline alkaloids.

\section{INTRODUÇÃO}

A família Rutaceae compreende cerca de 150 gêneros e 1600 espécies, amplamente distribuídas em regiões tropicais, subtropicais e temperadas do mundo, sendo mais abundante na América tropical, Sul da África e Austrália. ${ }^{1}$ No Brasil, a família está representada por cerca de 29 gêneros e 182 espécies, com algumas de importância medicinal, ecológica e econômica. ${ }^{1}$

$\mathrm{O}$ gênero Esenbeckia, pertencente à subtribo Pilocarpinae, possui cerca de 30 espécies distribuídas nas Américas, ${ }^{2}$ sendo que os principais centros de diversidade estão no sudeste do Brasil e no México. ${ }^{1}$ Destas, 22 espécies, incluindo E. grandiflora, tiveram sua constituição química investigada. Dentre os compostos isolados, alcaloides furoquinolínicos (E. alata, ${ }^{3}$ E. almawillia ${ }^{2,4}$ E. berlandieri, ${ }^{5}$ E. belizencis, ${ }^{6}$ E. conspecta,${ }^{7}$ E. flava,${ }^{5}$ E. febrifuga,${ }^{8}$ E. hieronimi,${ }^{9}$ E. leiocarpa,${ }^{10}$ E. litoralis,${ }^{5,11}$ E. pentaphylla ${ }^{12}$ E. pilocarpoides ${ }^{13} \mathrm{e}$ E. yaxhoob ${ }^{14}$ ), quinolinônicos (E. almawillia,${ }^{2,4,15-17}$ E. belizencis, ${ }^{6}$ E. flava,${ }^{5}$ E. hieronimi $i^{9}$ e E. leiocarpa ${ }^{10,18,19}$ E. pentaphylla ${ }^{12}$ e E. pilocarpoides ${ }^{13}$ ) e acridônicos (E. litoralis,${ }^{5}$ E. pentaphylla,${ }^{12}$ E. pilocarpoides $\left.^{13}\right)$, derivados alquil-, pirano- e furocumarínicos $\left(E\right.$. alata, ${ }^{3,20} E$. almawillia, ${ }^{4}$ E. berlandieri, ${ }^{5}$ E. conspecta,${ }^{7}$ E. flava,${ }^{5}$ E. febrifuga,${ }^{21}$ E. hieronimi, ${ }^{9}$ E. leiocarpa, ${ }^{10}$ E. litoralis, ${ }^{5,11}$ E. ovata, ${ }^{22}$ E. pentaphylla ${ }^{12}$ e E. yaxhoob ${ }^{23}$ ) e os limonoides (E. berlandieri, ${ }^{5}$ E. flava, ${ }^{5}$ E. febrifuga ${ }^{24}$ E. litoralis, ${ }^{5}$ E. hartmanni, ${ }^{5}$ E. ovata ${ }^{25}$ e E. yaxhoob ${ }^{23}$ ) constituem as classes predominantes e mais representativas sendo, portanto, consideradas como marcadores químicos para o gênero. Além destas, outras classes de ocorrência menos freqüente, tais como alcaloides indólicos (E. leiocarpa),${ }^{10,18}$ derivados fenilpropanoídicos (E. almawillia ${ }^{15,16}$ e E. leiocarpa ${ }^{10}$ ), terpenoides (E. almawillia, ${ }^{2,16}$ E. alata, ${ }^{3,26}$ E. belizencis, ${ }^{6}$ E. berlandieri $\mathrm{ssp}$. berlandieri, ${ }^{25}$ E. berlandieri ssp. acapulcensis, ${ }^{27}$ E. conspecta ${ }^{7}$ E. hieronimi,${ }^{9}$ E. litoralis,${ }^{11}$ E. nesiotica, ${ }^{28}$ E. ovata, ${ }^{22}$ E. stephani, ${ }^{29}$ E. yaxhoob ${ }^{14,23}$ e E. velutino$s a^{25}$ ), flavonoides (E. almawillia, ${ }^{2}$ E. berlandieri ssp. acapulcensis, ${ }^{30}$ E. pumila ${ }^{31,32}$ e $E$. yaxhoob $\left.{ }^{23}\right)$, poliprenois e acilfluoroglucinois $(E$. belizencis ${ }^{6}{ }^{E}$. nesiotica $\left.{ }^{28}\right)$, lignoides (E. alata, ${ }^{3,26}$ E. leiocarpa ${ }^{10} \mathrm{e} E$. yaxhoob ${ }^{23}$ ), cinamoilamidas (E. almawillia ${ }^{2}$ E. alata ${ }^{26}$ E. leiocarpa $^{18}$

*e-mail: anahjanuario@unifran.br e E. nesiotica $\left.{ }^{10,28}\right)$, bem como derivados cromanônico $\left(E . \operatorname{alata}^{26}\right)$ e antranilato (E. yaxhoob ${ }^{14,33}$ ) também foram isolados.

A espécie E. grandiflora, conhecida popularmente como limãozinho, gramicho, guarantã, cocão, cipó e garajaú, é uma planta arbórea com ampla distribuição no Brasil, especialmente em áreas de tabuleiros arenosos, restingas, matas costeiras e lugares úmidos, sendo que na região Sudeste ocorre principalmente em floresta latifoliada semidecídua. ${ }^{1}$ Por ser de pequeno porte e de uso ornamental, pode ser empregada no paisagismo. ${ }^{34}$ Do ponto de vista etnomedicinal, a literatura não registra qualquer atividade para esta espécie. No entanto, ensaios biológicos efetuados com extratos em clorofórmio e em acetato de etila das folhas revelaram atividade antibacteriana, ${ }^{35}$ além de atividade frente a larvas do $4^{\circ}$ instar do Aedes aegypti para o extrato em hexano das raízes $\left(\mathrm{CL}_{50} 108,23\right.$ ppm). ${ }^{36} \mathrm{O}$ estudo químico deste último resultou no isolamento dos derivados furocumarínicos pimpinelina e um dos seus produtos de fotodimerização, o 5,5',6,6'-tetrametoxi3,3', 4,4' -tetraidro-2H,2'H-3,3':4,4' -bi(furo[2,3-h][1]-benzopirano)2,2'-diona), ${ }^{36,37}$ swietenocumarina B, 7-metil-8-(3,3-dimetilalil) daphnetina, xantotoxina, 5-senecioilxantotoxina, isopimpinelina, 3-(1',1')-dimetilalilcolumbianetina e 5-(1'-hidroxi-isopentenil) bergapteno, além do sitosterol e da sitostenona.$^{36}$ Neste mesmo estudo, uma mistura constituída pela pimpinelina e pelo produto de fotodimerização $\left(\mathrm{CL}_{50} 45,77 \mathrm{ppm}\right)$, bem como de pimpinelina e de swietenocumarina $\mathrm{B}$ $\left(\mathrm{CL}_{50} 62,23 \mathrm{ppm}\right)$ também foram efetivos para larvas do A. aegypti. ${ }^{36}$ Além destes, o extrato em clorofórmio das raízes forneceu os alcaloides furoquinolínicos maculina, kokusaginina e flindersiamina, a quinolona 4-metoxi-1-metilquinolin-2-onae as furocumarinas xantotoxina, pimpinelina, 3-(1',1'-dimetil-alil) columbianetina.

De acordo com Kaastra e colaboradores,$^{38}$ a espécie E. grandiflora consiste de duas subespécies, E. grandiflora ssp. grandiflora e E. grandiflora ssp. brevipetiolata, que crescem no Sul e no Nordeste do Brasil, respectivamente. Os estudos químicos efetuados revelaram que ambas contêm os derivados cumarínicos cumarina $\mathrm{B}$, anisocumarina $\mathrm{H}$, imperatorina e (-)-heraclenol, ${ }^{38,39} 3$-(1',1'-dimetilalil) columbianetina, isopimpinelina, xantotoxina, pimpinelina e 5-senecioil-xantotoxina ${ }^{38,40}$, os flavonoides (-)-epigallocatequina, $3-O-\alpha-\mathrm{L}-$ rhamnopiranosilquercetina e 3-O- $\alpha$-L-rhamnopiranosilkaempferol e as di-hidrochalconas: F1, F2, F3, 2',4',6',4-tetra-hidroxi-3'-geranil- 
3-prenildi-hidrochalcona e 2',4',6',4-tetra-hidroxi-3'-geranildihidrochalcona, as quais diferem apenas no padrão de oxigenação do anel $\mathrm{B}{ }^{38-40}$ Além destes, alcaloides furoquinolínicos $\gamma$-fagarina, delbina, kokusaginina, skimmianina e flindersiamina também foram encontrados nas raízes de E. grandiflora ssp. brevipetiolata. ${ }^{38,40}$

O presente trabalho descreve o estudo químico de dois espécimes de E. grandiflora, coletados nas regiões Sul (Espécime I: folhas e galhos) e Nordeste (Espécime II: raízes, caule e folhas) do Brasil, visando contribuir para um maior conhecimento da sua química, bem como para a ampliação do perfil quimiotaxonômico do gênero Esenbeckia. Neste trabalho foram isolados das folhas do espécime I os triterpenos $\alpha$ - (1) e $\beta$-amirina (2), $\alpha$ - (3) e $\beta$-amirenonol (4), $3 \alpha$-hidroxi-ursan-12-ona (5), $3 \alpha$-hidroxi-12,13-epoxi-oleanano (6), o alcaloide furoquinolínico delbina (7), os alcaloides do tipo $\beta$-indolopiridoquinazolínico rutaecarpina (8) e 1-hidroxirutaecarpina (9), as cumarinas umbeliferona (10) e aurapteno (11) e os fitoesteroides sitosterol (12), estigmasterol (13), campesterol (14) e $3 \beta-O$ $\beta$ - $D$-glicopiranosilsitosterol (15); enquanto que dos galhos somente os compostos 1, 2 e 12 foram obtidos. Por outro lado, do espécime II foram isolados das raízes as furanocumarinas pimpinelina (16) e xantotoxina (17), o alcaloide $\beta$-indolopiridoquinazolínico euxiloforicina D (18) e o alcaloide furoquinolínico kokusaginina (19); do caule somente os compostos 12 e 19; ao passo que das folhas apenas os compostos 1, 2 e 15. De acordo com o levantamento efetuado, a ocorrência de alcaloides do tipo $\beta$-indolopiridoquinazolínicos no gênero Esenbeckia está sendo relatada pela primeira vez.

\section{RESULTADOS E DISCUSSÃO}

Os compostos $\alpha$ - (1) e $\beta$-amirina (2), $\alpha$ - (3) e $\beta$-amirenonol (4), $3 \alpha$-hidroxi-ursan-12-ona (5), $3 \alpha$-hidroxi-12,13-epoxi-oleanano (6), ${ }^{41}$ delbina (7), ${ }^{42}$ umbeliferona (10), ${ }^{43}$ aurapteno (11),${ }^{44}$ sitosterol (12), estigmasterol (13), campesterol (14), $3 \beta-O-\beta$ - $D$-glicopiranosilsitosterol (15) ${ }^{45}$ pimpinelina $(\mathbf{1 6}),{ }^{46}$ xantotoxina $(\mathbf{1 7})^{47}$ e kokusaginina $(\mathbf{1 9})^{48}$ foram identificados pela comparação dos dados espectrais obtidos com os dos respectivos compostos descritos na literatura.

O espectro de massas do composto 8 apresentou o pico correspondente ao íon molecular em $\mathrm{m} / \mathrm{z}, 287(60,3)$ e o espectro no IV revelou bandas de absorção que sugeriram a presença de deformação axial $\mathrm{N}-\mathrm{H}\left(3342 \mathrm{~cm}^{-1}\right)$ e de carbonila de amida $\left(1656 \mathrm{~cm}^{-1}\right)$. O espectro de RMN ${ }^{1} \mathrm{H}$ evidenciou sinais para sete hidrogênios aromáticos $[\delta 7,24$ (1H, ddd, $J=0,8 ; 7,2 ; 7,2 \mathrm{~Hz}, \mathrm{H}-10), 7,36$ (1H, ddd, $J=1,2 ; 7,2 ; 7,2$ $\mathrm{Hz}, \mathrm{H}-11), 7,43$ (1H, $J=1,2 ; 7,2 \mathrm{~Hz}, \mathrm{H}-3) ; 7,45$ (1H, dd, $J=0,8 ; 7,2$ Hz, H-12), 7,65 (2H, t, $J=7,2 \mathrm{~Hz}, \mathrm{H} 1, \mathrm{H}-9) ; 7,71$ (1H, dtd, $J=1,2$; $7,2 ; 8,0 \mathrm{~Hz}, \mathrm{H}-2)]$, além da presença de dois tripletos, integrados para dois hidrogênios cada, em $\delta 3,24$ e $\delta 4,59$ ( $J=7,0 \mathrm{~Hz}$ cada) e um singleto largo em $\delta 9,22(\mathrm{~N}-\mathrm{H})$ de um núcleo indólico. Observou-se ainda um duplo dupleto em $\delta 8,30(J=1,6 ; 8,0 \mathrm{~Hz})$, atribuído ao $\mathrm{H}-4$, desprotegido em decorrência da anisotropia do grupo carbonila. O experimento $\operatorname{COSY}{ }^{1} \mathrm{H}-{ }^{1} \mathrm{H}$ confirmou as interações entre $\mathrm{H}-3$ e $\mathrm{H}-4$, bem como entre os hidrogênios dos anéis $\mathrm{A}$ e $\mathrm{E}$ de um alcaloide do tipo $\beta$-indolopiridoquinazolínico.

O espectro de $\mathrm{RMN}{ }^{13} \mathrm{C}$ apresentou o sinal de um grupo carbonila em $\delta 161,6$ e sinais de dois carbonos em $\delta 41,1$ e $\delta 19,7$ compatíveis com um sistema do tipo $-\mathrm{CH}_{2} \mathrm{CH}_{2} \mathrm{NH}-\mathrm{C}=\mathrm{O}$. Os dados obtidos do espectro de $\mathrm{RMN}{ }^{13} \mathrm{C}$ de $\mathbf{8}$ (Tabela 1) estão em concordância com os descritos na literatura para a rutaecarpina, ${ }^{49}$ isolada pela primeira vez no gênero Evodia..$^{50}$

O espectro de massas do composto 9 revelou o pico referente ao íon molecular em $\mathrm{m} / \mathrm{z} 303$ (100\%) e o espectro de RMN ${ }^{1} \mathrm{H}$ mostrou-se semelhante ao do composto $\mathbf{8}$, exceto pela presença de sinais para sete hidrogênios aromáticos $[\delta 7,70(1 \mathrm{H}, \mathrm{dl}, J=7,9 \mathrm{~Hz}, \mathrm{H}-9), 7,56(2 \mathrm{H}$, $\mathrm{dt}, J=1,2 ; 7,9 \mathrm{~Hz}, \mathrm{H}-4$ e H-12), 7,30 (2H, t, $J=7,9 \mathrm{~Hz}, \mathrm{H}-3$ e H-11),
7,22 (1H, dd, $J=1,2$ e 7,9 Hz, H-2), 7,12 (1H, t, $J=7,9 \mathrm{~Hz}, \mathrm{H}-10)]$ e para um grupo hidroxila sugerido pela presença de um singleto largo em $\delta 9,33$. Também foram observados sinais para dois tripletos em $\delta$ $4,44(2 \mathrm{H}, J=6,8 \mathrm{~Hz})$ e em $\delta 3,02(2 \mathrm{H}, J=6,8 \mathrm{~Hz})$ referentes, respectivamente, ao H-7 e ao H-8, além de um grupo N-H em $\delta 11,54$ (sl). A localização do grupo hidroxila no anel $\mathrm{E}$ foi sugerida pela presença dos fragmentos em $\mathrm{m} / \mathrm{z}, 140(12)$ e $115(14,6)$ no espectro de massas. $\mathrm{O}$ experimento COSY ${ }^{1} \mathrm{H}-{ }^{1} \mathrm{H}$ auxiliou na atribuição dos sinais dos hidrogênios dos anéis A e E. A ausência de um singleto largo entre $\delta 12,0-15,0$ descartou a possibilidade do grupo hidroxila localizar-se em C-4. Este fato foi reforçado pela proteção observada para H-4 $(\delta$ 7,56). Estas análises permitiram identificar o composto 9 como sendo a 1-hidroxirutaecarpina, ${ }^{51,52}$ previamente isolada no gênero Euxylophora. ${ }^{3}$

$\mathrm{O}$ espectro de massas do composto $\mathbf{1 8}$ mostrou o pico correspondente ao íon molecular em $\mathrm{m} / \mathrm{z}, 377$ (100\%) e o espectro de absorção na região IV revelou além das bandas indicativas da presença de anel aromático $\left(1631,1548,1511,866\right.$ e $\left.823 \mathrm{~cm}^{-1}\right)$, a presença de grupo $\mathrm{N}-\mathrm{H}\left(3404 \mathrm{~cm}^{-1}\right)$ e de carbonila de amida $\left(1693 \mathrm{~cm}^{-1}\right)$. O espectro de $\mathrm{RMN}{ }^{1} \mathrm{H}$ evidenciou na região de aromáticos sinais cujos valores de deslocamentos químicos, multiplicidades e constantes de acoplamento estão compatíveis com a presença de um sistema $\mathrm{ABX}[\delta$ $7,20(1 \mathrm{H}, \mathrm{d}, J=2,3 \mathrm{~Hz}, \mathrm{H}-9), \delta 7,17$ (1H, dd, J = 8,8; 2,3 Hz, H-11) e $\delta 7,55(1 \mathrm{H}, \mathrm{d}, \mathrm{J}=8,8 \mathrm{~Hz}, \mathrm{H}-12)]$ e para dois hidrogênios isolados [ $\delta 7,38(1 \mathrm{H}, \mathrm{s}, \mathrm{H}-1)$ e $\delta 7,75(1 \mathrm{H}, \mathrm{s}, \mathrm{H}-4)]$. Adicionalmente foram observados sinais simples para três grupos metoxilas $(\delta 4,12, \delta 4,0 \mathrm{e}$ $\delta 3,88)$, um grupo $\mathrm{N}-\mathrm{H}[\delta 7,75(1 \mathrm{H}, \mathrm{s})]$ e para dois grupos metilenos do tipo $-\mathrm{CH}_{2} \mathrm{CH}_{2} \mathrm{~N}-[\delta 4,58(2 \mathrm{H}, \mathrm{t}, \mathrm{J}=6,7 \mathrm{~Hz}, \mathrm{H}-7)$ e $\delta 3,30(\mathrm{H}-8$, superposto ao sinal residual do solvente)]. Estes dados, associados às correlações observadas no espectro $\mathrm{COSY}{ }^{1} \mathrm{H}-{ }^{1} \mathrm{H}$ permitiram reconhecer o núcleo de um alcaloide do tipo $\beta$-indolopiridoquinazolínico. Os dados obtidos do espectro de $\mathrm{RMN}{ }^{13} \mathrm{C}$ (Tabela 1) permitiram identificar sinais cujos valores de deslocamentos químicos estão coerentes com a estrutura da euxiloforicina $\mathrm{D}$, isolada anteriormente em Euxylophora paraensis. ${ }^{53}$

\section{PARTE EXPERIMENTAL}

\section{Materiais e equipamentos}

Os espectros de RMN de ${ }^{1} \mathrm{H}$ e ${ }^{13} \mathrm{C}$ dos compostos de $\mathbf{1 - 1 5}$ foram obtidos em espectrômetro Brüker ARX-400 do Departamento de Química da Universidade Federal de São Carlos, operando a 400 e $100 \mathrm{MHz}$, respectivamente; enquanto que dos compostos 16-18 e 19 foram obtidos em espectrômetros Bruker AC-200 e Varian Gemini 300 , operando ${ }^{1} \mathrm{H}$ a 200 e $300 \mathrm{MHz}$ e ${ }^{13} \mathrm{C}$ a 50,3 e $75 \mathrm{MHz}$, respectivamente. Os espectros de massas dos compostos 8 e 9 foram obtidos no Departamento de Química da Universidade Federal de São Carlos, em um CG-EM da Hewlett-Packard, 5995 e os espectros de massas dos compostos 16-19 foram obtidos no Museu Paraense Emílio Goeldi, Belém-PA, em um CG-EM da Hewlett-Packard, 5890A/5988A. Os espectros de IV foram registrados em espectrofotômetro Perkin-Elmer FT-IR-1750, utilizando-se pastilhas de $\mathrm{KBr}$ contendo aproximadamente $1 \%$ de amostra.

\section{Materiais vegetais}

As folhas e galhos de um espécime identificado como E. grandiflora (Espécime I) foram coletadas no estado do Paraná. Paralelamente, folhas, caule e raízes de outro exemplar (Espécime II) foram coletados na área de restinga do município de Marechal Deodoro-AL e identificados pela botânica R. P. de L. Lemos, Instituto do Meio Ambiente do estado de Alagoas, Maceió-AL, onde um exemplar encontra-se catalogado (MAC-8426). 
Tabela 1. Dados de RMN dos compostos 8-9 $\left({ }^{1} \mathrm{H}: 400 ;{ }^{13} \mathrm{C}: 100 \mathrm{MHz}, \mathrm{CDCl}_{3}, \delta, \mathrm{Hz}\right)$ e $18\left({ }^{1} \mathrm{H}: 300 ;{ }^{13} \mathrm{C}: 75 \mathrm{MHz}, \mathrm{CD}, \mathrm{OD}, \delta, \mathrm{Hz}\right)$

\begin{tabular}{|c|c|c|c|c|c|c|}
\hline \multirow{2}{*}{ Posição } & \multicolumn{2}{|c|}{8} & \multicolumn{2}{|c|}{9} & \multicolumn{2}{|c|}{18} \\
\hline & $\delta_{\mathrm{C}}$ & $\delta_{\mathrm{H}}$ & $\delta_{\mathrm{C}}$ & $\delta_{\mathrm{H}}\left(\mathrm{DMSO}-d_{6}\right)$ & $\delta_{\mathrm{C}}$ & $\delta_{\mathrm{H}}$ \\
\hline 1 & 125,6 & $7,65(\mathrm{t}, 7,2)$ & 151,9 & & 101,0 & $7,38 \mathrm{~s}$ \\
\hline 2 & 134,3 & $\begin{array}{c}7,71 \\
(\mathrm{dtd}, 1,2 ; 7,2 ; 8,0)\end{array}$ & 116,2 & $\begin{array}{c}7,22 \\
(\mathrm{dd}, 1,2 ; 7,9)\end{array}$ & 150,2 & \\
\hline 3 & 126,6 & $7,43(\mathrm{t}, 1,2 ; 7,2)$ & 124,5 & $7,30(t, 7,6)$ & 147,6 & \\
\hline 4 & 127,2 & $\begin{array}{c}8,30 \\
(\mathrm{dd}, 1,6 ; 8,0)\end{array}$ & 127,3 & $\begin{array}{c}7,56 \\
(\mathrm{dd}, 1,2 ; 7,9)\end{array}$ & 115,4 & $7,75 \mathrm{~s}$ \\
\hline $4 \mathrm{a}$ & - & & 120,5 & & 111,7 & \\
\hline 5 & 161,6 & & 160,6 & & - & \\
\hline 7 & 41,1 & $4,59(t, 7,0)$ & 39,9 & $4,44(t, 6,8)$ & 43,6 & $4,58(t, 6,7)$ \\
\hline 8 & 19,7 & $3,24(t, 7,0)$ & 19,1 & $3,02(\mathrm{t}, 6,8)$ & 30,63 & $3,30^{*}$ \\
\hline $8 \mathrm{a}$ & 118,4 & & 116,4 & & 117,9 & \\
\hline $8 \mathrm{~b}$ & 127,4 & & 124,9 & & 123,6 & \\
\hline 9 & 120,6 & $7,65(t, 7,2)$ & 119,5 & $7,7(\mathrm{dl}, 7,9)$ & 101,4 & $7,20(\mathrm{~d}, 2,3)$ \\
\hline 10 & 120,1 & $\begin{array}{c}7,24 \\
(\mathrm{ddd}, 0,8 ; 7,2 ; 7,2)\end{array}$ & 119,6 & $7,12(\mathrm{t}, 7,9)$ & 155,5 & \\
\hline 11 & 126,2 & $\begin{array}{c}7,36 \\
\text { (ddd, } 1,2 ; 7,2 ; 7,2)\end{array}$ & 124,5 & $7,30(t, 7,9)$ & 108,7 & $\begin{array}{c}7,17 \\
(\mathrm{dd}, 8,8 ; 2,3)\end{array}$ \\
\hline 12 & 112,0 & $\begin{array}{c}7,45 \\
(\mathrm{dd}, 0,8 ; 7,2)\end{array}$ & 111,7 & $\begin{array}{c}7,56 \\
(\mathrm{dt}, 1,2 ; 7,9)\end{array}$ & 122,3 & $7,55(\mathrm{~d}, 8,8)$ \\
\hline $12 \mathrm{a}$ & 138,2 & & 138,2 & & 138,4 & \\
\hline $13 a$ & 127,2 & & 126,1 & & 135,5 & \\
\hline $13 \mathrm{~b}$ & 144,9 & & 143,2 & & 156,6 & \\
\hline 14 & 147,3 & & - & & 128,9 & \\
\hline $\mathrm{NH}$ & & $9,22 \mathrm{sl}$ & & $11,54 \mathrm{~s}$ & & - \\
\hline $\mathrm{OH}$ & & & & $9,33 \mathrm{~s}$ & & \\
\hline $\mathrm{MeO}-2$ & & & & & 56,2 & $4,12 \mathrm{~s}$ \\
\hline $\mathrm{MeO}-3$ & & & & & 57,6 & $3,88 \mathrm{~s}$ \\
\hline $\mathrm{MeO}-10$ & & & & & 57,0 & $4,0 \mathrm{~s}$ \\
\hline
\end{tabular}

* Encoberto pelo sinal do solvente.

\section{Isolamento dos constituintes químicos das folhas e galhos (Espécime I)}

Os materiais vegetais provenientes do espécime I [folhas e galhos $(717 \mathrm{~g})$ ] foram sucessivamente extraídos com éter de petróleo, $\mathrm{CH}_{2} \mathrm{Cl}_{2}$ e $\mathrm{MeOH}$,. Após secagem a vácuo foram obtidos os extratos em éter de petróleo (7,6 g), $\mathrm{CH}_{2} \mathrm{Cl}_{2}(12,2 \mathrm{~g})$ e $\mathrm{MeOH}$ (46,2 g). O extrato em éter de petróleo das folhas ( $7,63 \mathrm{~g})$ foi cromatografado em coluna de gel de sílica, usando-se como eluente misturas de hexano e acetona em polaridade crescente. As frações coletadas foram posteriormente purificadas para fornecer uma mistura dos compostos 1 e 2 (3,0 g), a mistura de 3 e 4 (2 mg), 5 (10 mg) e 6 (20 mg). O extrato em diclorometano $(12,2 \mathrm{~g})$ foi submetido a uma filtração cromatográfica em sílica sob vácuo usando-se sucessivamente como eluentes: hexano, $\mathrm{CH}_{2} \mathrm{Cl}_{2}$, hexano- $\mathrm{CH}_{2} \mathrm{Cl}_{2}(1: 1)$, AcOEt e $\mathrm{MeOH}$. A fração em hexano- $\mathrm{CH}_{2} \mathrm{Cl}_{2}$ 1:1 (2,6 g) após cromatografia em coluna forneceu $\mathbf{1 + 2}(500 \mathrm{mg}), \mathbf{1 1}(2 \mathrm{mg}), \mathbf{1 2}(12 \mathrm{mg})$ e uma mistura composta por 12,13 e 14 (30 mg). A fração em $\mathrm{CH}_{2} \mathrm{Cl}_{2}$ após ser cromatografada forneceu 7 (3 mg) e 9 (13 mg). Parte do extrato em metanol (20 g) foi submetida a uma partição $\mathrm{MeOH} / \mathrm{H}_{2} \mathrm{O} / \mathrm{CH}_{2} \mathrm{Cl}_{2}$. A fração metanólica (6 g) foi cromatografada em coluna de gel de sílica obtendo-se $\mathbf{1 + 2}$ (10 mg), 7 (2 mg), 8 (2 mg), 10 (2 mg) e $\mathbf{1 5}(2 \mathrm{mg})$. O extrato em éter de petróleo dos galhos $(1,3 \mathrm{~g})$ foi cromatografado em coluna de gel de sílica usando como eluente misturas de hexano e acetona em polaridade crescente obtendo-se $\mathbf{1 + 2}(5 \mathrm{mg})$ e $\mathbf{1 2}(6 \mathrm{mg})$.

\section{Isolamento dos constituintes químicos das folhas, caule e raízes} (Espécime II)

Os materiais vegetais oriundos do espécime II [folhas (500 g), caule $(800$ g) e raízes $(1700$ g) ], após secagem a temperatura ambiente e trituração, foram individualmente extraídos em aparelho de Soxhlet com EtOH 90\%. Após concentração das soluções em evaporador rotatório, os extratos obtidos [folhas (140,2 g), caule (50,4 g) e raízes $(64,0 \mathrm{~g})$ ] foram suspensos em solução $\mathrm{MeOH}-\mathrm{H}_{2} \mathrm{O}$ (3:2) e extraídos sucessivamente com hexano, $\mathrm{CHCl}_{3}$ e AcOEt. Parte do material 
proveniente da fração em $\mathrm{CHCl}_{3}$ das folhas $(30,0 \mathrm{~g})$, após sucessivos fracionamentos cromatográficos em gel de sílica (70-230 mesh) utilizando-se misturas binárias de $\mathrm{C}_{6} \mathrm{H}_{14}-\mathrm{CHCl}_{3}$ e $\mathrm{CHCl}_{3}$-AcOEt em proporções crescentes de polaridade forneceu uma mistura constituída de $\mathbf{1}+\mathbf{2}(110 \mathrm{mg})$ e o composto $\mathbf{1 5}$ (45 mg).

A fração em $\mathrm{CHCl}_{3}(19,8 \mathrm{~g})$, procedente da partição do extrato em EtOH do caule, após sucessivos fracionamentos cromatográficos em gel de sílica (70-230 mesh) e misturas binárias de eluentes de diferentes polaridades $\left(\mathrm{C}_{6} \mathrm{H}_{14}\right.$ - $\mathrm{AcOEt}$ e $\mathrm{CHCl}_{3}$-AcOEt$)$ resultou no isolamento dos compostos 12 (20 mg) e 19 (15 mg).

A fração em AcOEt $(5,21 \mathrm{~g})$, proveniente da partição do extrato em EtOH das raízes, foi fracionada em gel de sílica com misturas de $\mathrm{CHCl}_{3}$-AcOEt em proporções crescentes de polaridade. Os materiais oriundos das subfrações reunidas 1-10 (0,02 g) e 11-16 (0,02 g), após sucessivas recristalizações com $\mathrm{C}_{6} \mathrm{H}_{14}-\mathrm{CHCl}_{3}$ forneceram os compostos $\mathbf{1 6}(9 \mathrm{mg})$ e $\mathbf{1 7}(12 \mathrm{mg})$, respectivamente. O material resultante das subfrações mais polares, 106-110 (3,75 g), após fracionamento cromatográfico em gel de sílica (70-230 mesh, $\mathrm{CHCl}_{3}-\mathrm{MeOH}$ em proporções crescentes de polaridade), cromatotron em $\mathrm{CHCl}_{3}-\mathrm{MeOH}$ (9:1) e CCDP (gel de sílica, $\mathrm{CHCl}_{3}-\mathrm{MeOH}$ 95:5) forneceu 18 (8 mg).<smiles>[R]C(=O)[Y3]([H])=O</smiles><smiles>[R][R]([R])=O</smiles><smiles>C[C@H]1C(I)CC[C@@]2(C)CC[C@]3(C)C(C(=O)CC4[C@@]5(C)CC[C@@H](O)C(C)(C)C5CC[C@]43C)C12</smiles>

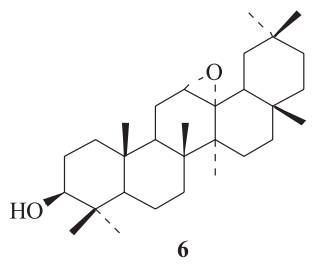<smiles>[R20]c1ccc2ccc(=O)oc2c1</smiles>

10: $\mathrm{R}=\mathrm{H}$ 11: $\mathrm{R}=$ Gerani<smiles>COc1c2c(c3ccoc3c1OC)CC(=O)C=C2</smiles>

16

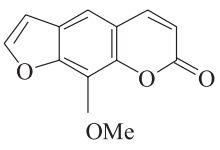

17
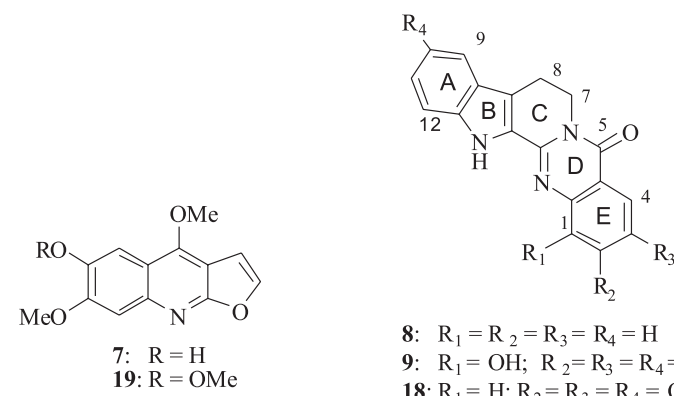

8: $\mathrm{R}_{1}=\mathrm{R}_{2}=\mathrm{R}_{3}=\mathrm{R}_{4}=\mathrm{H}$ 9: $\mathrm{R}_{1}=\mathrm{OH} ; \mathrm{R}_{2}=\mathrm{R}_{3}=\mathrm{R}_{4}=\mathrm{H}$ 18: $\mathrm{R}_{1}=\mathrm{H} ; \mathrm{R}_{2}=\mathrm{R}_{3}=\mathrm{R}_{4}=\mathrm{OMe}$

Figura 1.

\section{Rutaecarpina $(\mathbf{8})$}

Sólido amorfo. $[\alpha]_{\mathrm{D}}+67,7\left(25^{\circ} \mathrm{C} ; \mathrm{MeOH} ; \mathrm{c} 0,011\right)$. UV $(\mathrm{MeOH}$;

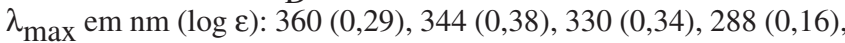

$276(0,16)$. IV (KBr,v $\left.v_{\max } \mathrm{em} \mathrm{cm}^{-1}\right)$ : 3342, 2922, 2851, 1739, 1656, 1601, 1466, 1328, 1230, 1085, 730, 691. EM m/z (int. rel.): 287 (60,3), $286(82,6), 168(11,6), 143(14,1), 129(31), 115(40,8)$, 77 (77), 69 (49), 57 (100), 55 (83). RMN RMN ( ${ }^{1} \mathrm{H}: 400 ;{ }^{13} \mathrm{C}: 100$ $\mathrm{MHz}, \mathrm{CDCl}_{3}$ ): Tabela 1 .

\section{1-Hidroxirutaecarpina $(\mathbf{9})$}

Sólido amarelado em forma de agulhas, p.f. $293,1-300,2{ }^{\circ} \mathrm{C}$. EM $\mathrm{m} / \mathrm{z}$ (int. rel.): 303 (100), 302 (98,2), 140 (12), $129(15,5), 115(14,6)$, 97 (11,4), 85 (10,2), 77 (14,7), $73(19,6), 69$ (17,8), 57 (19,7), 55 (20,1). RMN $\left({ }^{1} \mathrm{H}: 400 ;{ }^{13} \mathrm{C}: 100 \mathrm{MHz}, \mathrm{CDCl}_{3}\right)$ : Tabela 1 .

\section{Euxiloforicina $D(\mathbf{1 8})$}

Sólido amorfo, p.f. $293,3-294{ }^{\circ} \mathrm{C}$. IV (KBr) $\mathrm{cm}^{-1}$ : 3404, 2923, 2849 , 1693, 1631, 1548, 1511, 1443, 1396, 1321, 1304, 1263, 1212, 1015, 823, 764. EM m/z (int. rel.): 377 (100), 376 (28), 375 (21), 363 (36), 362 (62), 361 (17), 348 (18), 346 (13), 334 (19), 332 (12), 319 (10), 318 (15), 306 (3), 188 (21), 181 (17), 178 (11), 177 (13), 167 (15), 166 (11), 159 (12), 137 (22). RMN ( $\left.{ }^{1} \mathrm{H}: 300 ;{ }^{13} \mathrm{C}: \mathrm{MHz}, \mathrm{CD}_{3} \mathrm{OD}\right)$ : Tabela 1.

\section{CONCLUSÕES}

A investigação química de E. grandiflora permitiu o isolamento e identificação dos alcaloides 1-hidroxirutaecarpina, rutaecarpina e euxiloforicina D. Sendo este o primeiro relato da presença de alcaloides do tipo indolopiridoquinazolínico neste gênero.

$\mathrm{O}$ alcaloide rutaecarpina foi isolado anteriormente na família Rutaceae nos gêneros Evodia, Hortia, Zanthoxylum, Phellodendron, Tetradium, Spiranthera, Vepris, Metrodorea, Bouchardatia e Fagara da família Rutaceae e, só mais recentemente foi também isolado nas famílias Taxus chinensis (Texaceae) e Winchia calophylla (Apocynaceae) ${ }^{54}$ Por sua vez, a 1-hidroxirutaecarpina foi isolada previamente em Euxylophora paraënsis, Vepris louissi, Bouchardatia neurococca, Tetradium glabrifolium, Zanthoxylum integrifoliolum, Z. pistaciiflorum e Spiranthera odoratissima. Segundo estudos quimiossistemáticos realizados até o momento, Zanthoxylum é o único gênero de Rutaceae capaz de produzir a rutaecarpina juntamente com seus derivados, sendo este fato também inédito no gênero Esenbeckia. Em contrapartida, nos gêneros Euxylophora, Vepris e Leptotyrsa são encontrados diversos derivados da rutaecarpina, sem nenhum traço de rutaecarpina. ${ }^{54}$

\section{AGRADECIMENTOS}

Ao CNPq e FAPEAL pelo apoio financeiro concedido, ao CNPq pela bolsa de Pós-Graduação de A. H. Januário, à CAPES pela bolsa de J. J. de B. Silva, ao Instituto de Química da USP e Departamento de Química da Universidade Federal do Pará, pelos espectros de RMN a 200 e $300 \mathrm{MHz}$, respectivamente, e ao Museu Paraense Emílio Goeldi, Belém-PA, pelos espectros de EM.

\section{REFERÊNCIAS}

1. Melo, M. F. F.; Zickel, C. S.; Acta Bot. Bras. 2004, 18, 73.

2. Barros-Filho, B. A.; Nunes, F. M.; Oliveira, M. C. F.; Andrade-Neto, M.; Mattos M. C.; Quim. Nova 2007, 30, 1589.

3. Cuca-Suarez, L. E.; Barrera, C. A. C.; Biochem. Syst. Ecol. 2007, 35, 386.

4. Oliveira, F. M.; Santana, A. E. G.; Conserva, L. M.; Maia, J. G.; Guilhon, G. M. P.; Phytochemistry 1996, 41, 647.

5. Dreyer, D. L.; Phytochemistry 1980, 19, 941.

6. Rios, M. Y.; Delgado, G.; J. Nat. Prod. 1992, 55, 1307.

7. Rios, M. Y.; Rosas-Alonso, E.; Aguilar-Guadarrama, A. B.; Biochem. Syst. Ecol. 2002, 30, 367. 
8. Vitagliano, J. C.; Comin, J.; An. Asoc. Argent. 1970, 58, 59.

9. Delle Monache, F.; Trani, M.; Yunes, R. A.; Falkenberg, D.; Fitoterapia 1995, 66, 474.

10. Monache, F. D.; Monache, G. D.; E. De Moraes e Souza, M. A.; Cavalcanti, M. S.; Chiappeta, A.; Gazz. Chim. Ital. 1989, 119, 435.

11. Rios, M. Y.; Guadarrama, A. B.; Delgado, G.; Biochem. Syst. Ecol. 2002, 30, 977.

12. Simpson, D. S.; Jacobs, H.; Biochem. Syst. Ecol. 2005, 33, 841.

13. Bevalot, F.; Fournet, A.; Moretti, C.; Vaquette, J.; Planta Med. 1984, 50, 522.

14. Aguilar-Guadamarra, A. B.; Rios, M. Y.; Planta Med. 2004, 70, 85.

15. Guilhon, G. M. S. P.; Baetas, A. C. S.; Maia, J. G. S.; Conserva, L. M.; Phytochemistry 1994, 37, 1193.

16. Barros-Filho, B. A.; Fátima, M. N. A.; De Oliveira, M. C. F.; Mafezoli, J.; Andrade-Neto, M.; Silveira, E. R.; Pirani, J. R.; Biochem. Syst. Ecol. 2004, 32, 817 .

17. Nunes, F. M.; Barros-Filho, B. A.; de Oliveira, M. C. F.; de Mattos, M. C.; Andrade-Neto, M.; Barbosa, F. G.; Mafezoli, J.; Montenegro, R. C.; Pessoa, C.; de Moraes, M. O.; Costa-Lotufo, L. V.; Galeti, F. C. S.; Silva, C. L.; de Souza, A. O.; Nat. Prod. Commun. 2006, 1, 313.

18. Delle Monache, F.; Di Benedetto, R.; De Moraes e Souza, M. A.; Sandor, P.; Gazz. Chim. Ital. 1990, 120, 387.

19. Nakatsu, T.; Johns, T.; Kubo, I.; Milton, K.; Sakai, M.; Chatani, K.; Saito, K.; Yamagiwa, Y.; Kamikawa, T.; J. Nat. Prod. 1990, 53, 1508.

20. Garcia-Beltran, O. J.; Cuca-Suarez, L. E.; Actualidades Biologicas (Medellín, Colombia) 2005, 27, 71. (CA 145:99555x).

21. Napolitano, H. B.; Silva, M.; Ellena, J.; Rodrigues, B. D. G.; Almeida, A. L. C.; Vieira, P. C.; Oliva, G.; Thiemann. O. H.; Braz. J. Med. Biol. Res. 2004, 37, 1847.

22. Rios, M. Y.; Delgado, G.; Biochem. Syst. Ecol. 2002, 30, 697.

23. Mata, R.; Macias, M. L.; Rojas, I. S.; Lotina-Hennsen, B.; Toscano, R. A.; Anaya, A. L.; Phytochemistry 1998, 49, 441.

24. Vitagliano, J. C.; Comin, J.; An. Asoc. Quím. Argent. 1970, 58, 273.

25. Cano, A.; Bucio, J. L.; Espinoza, M.; Ruiz-Cancino, A.; Rev. Soc. Quim. Méx. 2000, 44, 155.

26. Garcia-Beltran, O. J.; Cuca-Suarez, L. E.; Rev. Colomb. Quim. 2003, 32, 23.

27. Cano, A.; Bucio, J. L.; Espinoza, M.; Ramos, C. H.; Delgado, G.; J. Mex. Chem. Soc. 2006, 50, 71.

28. Rios, M. Y.; Delgado, G.; Phytochemistry 1992, 31, 3491.

29. Rios, M. Y.; Aguilar-Guadarrama, A. B.; Biochem. Syst. Ecol. 2002, 30, 1006.

30. Cano, A.; Bucio, J. L.; Espinoza, M.; Ruiz-Cancino, A.; Rev. Soc. Quim. Méx. 2000, 44, 155.
31. Kubo, I.; Vieira, P. C.; Fukuhara, K.; J. Liq. Chromatog. 1990, 13, 2441.

32. Kubo, I.; J. Chromatog. 1991, 538, 187.

33. Sharma, S. D.; Sharma, M. L.; Rathee, R.; J. Indian Chem. Soc. 2006 , 83,1158 .

34. Da Silva, L. L.; Paoli, A. A. S.; Rev. Bras. Sementes 2006, $28,1$.

35. Novais, T. S.; Costa, J. F. O.; David, J. P. L.; David, J. M.; Queiroz, L. P.; França, F.; Giullietti, A. M.; Soares, M. B. P.; Santos, R.R.; Rev. Bras. Farmacog. 2003, 14, 5 .

36. Oliveira, P. E. S.; Conserva, L. M.; Brito, A. C.; Lemos, R. P. L.; Pharm. Biol. 2005, 43, 53.

37. De Oliveira, P. E. S.; Conserva, L. M.; De Simone, C. A.; Pereira, M. A.; Malta, V. R. S.; Imbroisi, D. O.; Acta Crystallog. Sect. C-Crystal Struct. Comm. 2004, 60, O900.

38. Kaastra, R. C.; Pilocarpineae (Rutaceae). Flora Neotropica Monography 33, New York Botanical Garden: New York, 1982.

39. Trani, M.; Delle Monache, F.; Delle Monache, G.; Yunes, R. A.; Falkenberg, D. B.; Gazz. Chim. Ital. 1997, 127, 415.

40. Trani, A.; Carbonetti, A.; Delle Monache, G.; Delle Monache, F.; Fitoterapia 2004, 75, 99.

41. Mahato, S. B.; Kundu, A. P.; Phytochemistry 1994, 37, 1517.

42. Bhattacharyya, J.; Serur, L. M.; Heterocycles 1981, 16, 371.

43. Sankar, S. S.; Gilbert, R. D.; Fornes, R. E.; Org. Magn. Res. 1982, 19 , 222.

44. Abulrob, A-N.; Suller, M. T. E.; Gumbleton, M.; Simons, C.; Russell, A. D.; Phytochemistry 2004, 65, 3021.

45. Tandon, M.; Shukla, Y. N.; Thakur, R. S.; Phytochemistry 1990, 29, 2957.

46. Souri, E.; Farsam, H.; Sarkheil, P.; Ebadi, F.; Pharm. Biol. 2004, 42, 396.

47. Sajjadi, S. E.; Noroozi, P.; Res. Pharma. Sci. 2007, 2,13

48. Pusset, J.; Lopez, J. L.; Pais, M.; Al Neirabeyeh, M.; Veillon, J. M.; Planta Med. 1991, 57, 153.

49. Bergman, J.; Bergman, S.; J. Org. Chem. 1985, 50, 1246.

50. Kamikado, T.; Murakoshi, S.; Tamura, S.; Agric. Biol. Chem. 1978, 42, 1515

51. Danieli, B.; Farachi, C.; Palmisano, G. A.; Phytochemistry 1976, 15, 1095.

52. Ayafor, J. F.; Sondengam, B. L.; Ngadjui, B. T.; Phytochemistry 1982, $21,2733$.

53. Danieli, B.; Palmisano, G.; Russo, G.; Ferrari, G.; Phytochemistry 1973, 12,2521

54. Lee, S. H., Son, J-K.; Jeong, B. S.; Jeong, T-C.; Chang, H. W.; Lee, E-S.; Jahng, Y.; Molecules 2008, 13, 272. 